\title{
MULTIPLE POSITIVE SOLUTIONS OF RESONANT AND NON-RESONANT NON-LOCAL FOURTH-ORDER BOUNDARY VALUE PROBLEMS
}

\author{
J. R. L. WEBB \\ School of Mathematics and Statistics, University of Glasgow, Glasgow G12 8QW, UK \\ e-mail: jeffrey.webb@glasgow.ac.uk \\ and M. ZIMA \\ Institute of Mathematics, University of Rzeszów, 35-959 Rzeszów, Poland \\ e-mail:mzima@univ.rzeszow.pl
}

(Received 19 March 2011; accepted 15 August 2011)

Abstract. We study the existence of positive solutions for equations of the form $u^{(4)}(t)-\omega^{4} u(t)=f(t, u(t))$, a.e. $t \in(0,1)$,

where $0<\omega<\pi$, subject to various non-local boundary conditions defined in terms of the Riemann-Stieltjes integrals. We prove the existence and multiplicity of positive solutions for these boundary value problems in both resonant and non-resonant cases. We discuss the resonant case by making a shift and considering an equivalent nonresonant problem.

2000 Mathematics Subject Classification. Primary 34B10, secondary 34B15, $34 \mathrm{~B} 18$.

1. Introduction. In the paper we are interested in the existence of positive solutions of some non-local boundary value problems (BVPs) for fourth-order equations of the form

$$
u^{(4)}(t)-\omega^{4} u(t)=f(t, u(t)), \quad \text { a.e. } t \in(0,1),
$$

for some constant $\omega \in(0, \pi)$, subject to the following non-local boundary conditions (BCs),

$$
u(0)=\beta_{1}[u], u^{\prime \prime}(0)+\beta_{2}[u]=0, u(1)=\beta_{3}[u], u^{\prime \prime}(1)+\beta_{4}[u]=0,
$$

where each $\beta_{i}[u]$ is a linear functional on $C[0,1]$, that is it is given by a Riemann-Stieltjes integral,

$$
\beta_{i}[u]=\int_{0}^{1} u(s) d B_{i}(s) .
$$

Since some of the $\beta_{i}$ can be zero, while others are not, this covers many BCs.

Support from the Glasgow Journal Trust for Mirosława Zima is gratefully acknowledged. 
For (1.1) we consider cases where $f(t, u)$ is not positive for all positive $u$ but is such that $f(t, u)+k^{4} u \geq 0$ for $u \geq 0$ for a suitable constant $k \in(0, \omega)$. One important motivation is that the original problem (1.1) with the BCs (1.2) may be at resonance, that is, $\lambda=0$ is an eigenvalue of the linear problem $u^{(4)}-\omega^{4} u=\lambda u$ with the given BCs. In such a case we can consider the equivalent problem, which is of the same type as the original one,

$$
u^{(4)}(t)-\widetilde{\omega}^{4} u(t)=\tilde{f}(t, u(t))
$$

where $\widetilde{\omega}^{4}:=\omega^{4}-k^{4}, \widetilde{f}(t, u):=f(t, u)+k^{4} u$, with same BCs. We will show that, under natural conditions, this perturbed problem is non-resonant.

The fact that we consider BCs involving functions of bounded variation, equivalently signed measures, with no extra effort, is one of the features of our work. Some kind of positivity on the functionals $\beta_{i}$ is needed in order to have positive solutions, a solution $u$ will satisfy $\beta_{i}[u] \geq 0$ but we do not suppose that $\beta_{i}[u] \geq 0$ for all $u \geq 0$. Most other works have considered only special cases with positivity conditions assumed, our results are also new even for the positive case.

These type of $\mathrm{BCs}$, linear functionals on $C[0,1]$, are quite natural and have the advantage of including multipoint $\mathrm{BCs}$, where $\beta_{i}[u]:=\sum_{j=1}^{m} \beta_{i j} u\left(\eta_{j}\right), 0<\eta_{1}<\eta_{2}<$ $\cdots<\eta_{m}<1$ and integral BCs, where $\beta_{i}[u]:=\int_{0}^{1} b_{i}(s) u(s) d s$, and sum of these, in a single framework.

Multipoint BCs for second-order equations have been extensively studied in recent years when all the coefficients $\beta_{i j}$ are positive or have the same sign (see for example $[1,5,6,10,17])$. There are also works on fourth- and higher order problems, see for example [2-4, 8, 28, 29]. Our work covers more general BCs, and for the special case of multipoint BCs some coefficients of both signs are allowed.

We use fixed point index theory, based on the methods developed in [26, 28]. The fourth-order equation when $\omega=0$ with a variety of BCs, and with one BC of the non-local type that we study here, has been studied, with similar methods, in detail in [29] in the non-resonant case.

Many papers dealing with resonant problems use Mawhin's coincidence degree theory [20], for example $[\mathbf{5}, \mathbf{1 1}, \mathbf{1 2}, \mathbf{1 6}, \mathbf{1 9}]$ or an extension of this $[\mathbf{1 0}, \mathbf{2 1}]$. A paper using global bifurcation is by Ma and Chen [18]. Using a shift argument was done for second-order problems in [7, 31], some other work where using a shift argument proved useful is [9]. An advantage of using a shift is that we can prove existence of multiple positive solutions in the resonance case. An example possessing one positive solution and an example with two positive solutions are given at the end of the paper.

Our study begins with the well-known case where $\beta_{i} \equiv 0$, we call this the local problem. This problem is a model of the steady states of a deflected elastic beam with simply supported ends, also called hinged ends; the displacement and bending moments at each end are zero. The interpretation of the non-local BCs are as feedback controls, measurements of the displacement are made along parts of the beam and controllers at the endpoints make adjustments according to these measurements.

The local problem with both possible signs

$$
u^{(4)}(t) \pm \omega^{4} u(t)=f(t, u(t)), \quad u(0)=0, u^{\prime \prime}(0)=0, u(1)=0, u^{\prime \prime}(1)=0,
$$

has been studied in detail in [2], using an anti-maximum principle and utilising upper and lower solutions. The corresponding problem with clamped end BCs is similarly studied in [3]; non-local BCs are not treated in those papers. 
A standard approach to studying positive solutions of (1.1) and (1.2) in the nonresonant case is to find the corresponding Green's function $G$ and seek solutions as fixed points of the integral operator

$$
S u(t):=\int_{0}^{1} G(t, s) f(s, u(s)) d s
$$

in the cone $P=\{u \in C[0,1]: u \geq 0\}$ of non-negative functions in the space $C[0,1]$ of continuous functions with the usual supremum norm. Certain properties of the Green's function are needed to give a good existence theory, a useful one, essentially as used by Lan and Webb [15], is that there exist positive functions $\Phi, c$ such that

$$
c(t) \Phi(s) \leq G(t, s) \leq \Phi(s), \quad \text { for } 0 \leq t, s \leq 1 .
$$

The Green's function for the local problem is well known; we will give it and some of its properties later in the paper, in particular we will show that it satisfies (1.6). Our approach to the problem with several BCs of non-local type uses the theory developed in [28]. The non-local problem, (1.1) and (1.2), is regarded as a perturbation of the local problem. That paper gave a simple derivation of an explicit formula for the Green's function of the non-local problem once the Green's function for the local problem is known. In particular, a simple method, which avoids long calculations, shows that (1.6) also holds for the non-local Green's function.

We give results on the existence of multiple positive solutions and also results on the non-existence of a positive solution, which shows that some of our hypotheses are sharp.

2. Integral operators. We will study the existence of positive solutions of the fourth-order nonlinear BVP

$$
u^{(4)}(t)-\omega^{4} u(t)=f(t, u(t))
$$

with $0<\omega<\pi$, subject to the BCs

$$
u(0)=\beta_{1}[u], \quad u^{\prime \prime}(0)+\beta_{2}[u]=0, \quad u(1)=\beta_{3}[u], \quad u^{\prime \prime}(1)+\beta_{4}[u]=0,
$$

where $\beta_{i}[u]$ are linear functionals on the space $C[0,1]$, that is these are given by the Riemann-Stieltjes integrals,

$$
\beta_{i}[u]=\int_{0}^{1} u(s) d B_{i}(s)
$$

with signed measures, that is $B_{i}$ are functions of bounded variation. Although we do not suppose that $\beta_{i}[u] \geq 0$ for all $u \in P$, we write $\mathrm{BCs}$ in the form given above because we will find positive solutions that satisfy $\beta_{i}[u] \geq 0$. Here $u$ is called positive if $u \in P \backslash\{0\}$.

We use the theory developed by Webb and Infante in [28] to study the existence of positive solutions for (2.1) and (2.2) in the non-resonant case. We seek solutions as fixed points of the integral operator

$$
S u(t):=\int_{0}^{1} G(t, s) f(s, u(s)) d s,
$$


where $G$ is the Green's function. This integral equation was studied in [28] under the following conditions imposed on $G$ and $f$ :

$\left(C_{1}\right) G \geq 0$ is measurable, and for every $\tau \in[0,1]$ we have

$$
\lim _{t \rightarrow \tau}|G(t, s)-G(\tau, s)|=0 \quad \text { for a.e. } s \in[0,1]
$$

$\left(C_{2}\right)$ There exists a subinterval $[a, b] \subseteq[0,1]$, a non-negative function $\Phi \in L^{1}(0,1)$, with $\int_{a}^{b} \Phi(s) d s>0$, and a constant $c_{0}=c_{0}(a, b) \in(0,1]$ such that

$$
\begin{gathered}
G(t, s) \leq \Phi(s) \quad \text { for } t \in[0,1] \text { and a.e. } s \in[0,1] \\
G(t, s) \geq c_{0} \Phi(s) \quad \text { for } t \in[a, b] \text { and a.e. } s \in[0,1] .
\end{gathered}
$$

$\left(C_{3}\right) f:[0,1] \times[0, \infty) \rightarrow[0, \infty)$ satisfies the Carathéodory conditions, that is $f(\cdot, u)$ is measurable for each fixed $u \in[0, \infty)$ and $f(t, \cdot)$ is continuous for almost every $t \in[0,1]$, and for each $r>0$, there exists $\phi_{r} \in L^{\infty}[0,1]$ such that

$$
f(t, u) \leq \phi_{r}(t) \text { for all } u \in[0, r] \text { and almost all } t \in[0,1] .
$$

It is often convenient to prove the following type of inequality:

$\left(C_{2}^{\prime}\right) \quad c(t) \Phi(s) \leq G(t, s) \leq \Phi(s), \quad$ for $0 \leq t, s \leq 1$,

for a function $c \in P \backslash\{0\}$, which establishes the inequality in $\left(C_{2}\right)$ when $c(t) \geq c_{0}>0$ on $[a, b]$. When $c(t)>0$ on $(0,1),[a, b]$ may be an arbitrary subset of $(0,1)$, so can be chosen to suit the problem and can give weaker conditions. In particular, often the interval can be chosen to minimise a constant called $M=M(a, b)$ that occurs in the theory.

In [28] Webb and Infante gave a new method of determining the Green's function for many non-local BVPs of arbitrary order that avoids long calculations.

The idea used in $[\mathbf{2 6}, \mathbf{2 8}]$ is to consider solution of the non-local problem as perturbations from the non-local problem and to seek fixed points of the following operator

$$
T u(t)=B u(t)+S_{0} u(t):=\sum_{i=1}^{4} \beta_{i}[u] \gamma_{i}(t)+\int_{0}^{1} G_{0}(t, s) f(s, u(s)) d s,
$$

where $G_{0}$ is the Green's function for the local problem (when all $\beta_{i}$ are 0 ), and $\gamma_{i}$ are solutions of $\gamma^{(4)}(t)-\omega^{4} \gamma(t)=0$ with a simple non-zero BC. In our case, these are solutions of

$$
\gamma^{(4)}(t)-\omega^{4} \gamma(t)=0
$$

under each of the local BCs:

$$
\begin{aligned}
& \gamma(0)=1, \gamma^{\prime \prime}(0)=0, \gamma(1)=0, \gamma^{\prime \prime}(1)=0, \\
& \gamma(0)=0, \gamma^{\prime \prime}(0)=-1, \quad \gamma(1)=0, \gamma^{\prime \prime}(1)=0, \\
& \gamma(0)=0, \gamma^{\prime \prime}(0)=0, \gamma(1)=1, \gamma^{\prime \prime}(1)=0, \\
& \gamma(0)=0, \gamma^{\prime \prime}(0)=0, \gamma(1)=0, \gamma^{\prime \prime}(1)=-1 .
\end{aligned}
$$


The solutions are readily found and are

$$
\begin{aligned}
& \gamma_{1}(t)=\sin (\omega(1-t)) /(2 \sin (\omega))+\sinh (\omega(1-t)) /(2 \sinh (\omega)), \\
& \gamma_{2}(t)=\sin (\omega(1-t)) /\left(2 \omega^{2} \sin (\omega)\right)-\sinh (\omega(1-t)) /\left(2 \omega^{2} \sinh (\omega)\right), \\
& \gamma_{3}(t)=\sin (\omega t) /(2 \sin (\omega))+\sinh (\omega t) /(2 \sinh (\omega)), \\
& \gamma_{4}(t)=\sin (\omega t) /\left(2 \omega^{2} \sin (\omega)\right)-\sinh (\omega t) /\left(2 \omega^{2} \sinh (\omega)\right),
\end{aligned}
$$

for (2.6) and (2.7), (2.6)-(2.8), (2.6)-(2.9) and (2.6)-(2.10), respectively. Clearly, the functions $\gamma_{i}$ are continuous, positive on $(0,1)$ (since $\omega \in(0, \pi)$ ) and linearly independent.

In fact, we can consider

$$
T u(t)=B u(t)+S_{0} u(t)=\sum_{i=1}^{N} \beta_{i}[u] \gamma_{i}(t)+\int_{0}^{1} G_{0}(t, s) f(s, u(s)) d s,
$$

where $N$ may be any integer number between 0 and 4, that is we can, and should, omit in the calculations those $\beta_{i}[u]$ that are identically zero. Then a non-zero fixed point of (2.12) in the cone $P$ is a positive solution of the BVP (2.1) and (2.2).

Let $[B]$ denote the $N \times N$ matrix $[B]:=\left[b_{i j}\right]$, where $b_{i j}=\beta_{i}\left[\gamma_{j}\right]$, and let

$$
\mathcal{G}_{i}(s):=\int_{0}^{1} G_{0}(t, s) d B_{i}(t)
$$

where $G_{0}$ is the Green's function for the local problem (when all $\beta_{i}$ are 0), which is given explicitly in (2.21). When we need to stress the dependence on $\omega$ we will write $\left[B_{\omega}\right]$.

To study positive fixed points we make the following additional assumptions:

$\left(C_{4}\right)$ For each $i, B_{i}$ is a function of bounded variation and $\mathcal{G}_{i}(s) \geq 0$ for a.e. $s \in[0,1]$.

$\left(C_{5}\right)$ The $N \times N$ matrix $[B]:=\left[b_{i j}\right]$ with $b_{i j}=\beta_{i}\left[\gamma_{j}\right]$ is non-negative, that is $b_{i j} \geq 0$, and the spectral radius of $[B]$, denoted by $r([B])$, satisfies $r([B])<1$.

It is shown in [28] that the operator $B$ and the matrix $[B]$ are closely related, for example $B$ and $[B]$ have equal spectral radii, $r(B)=r([B])$, in particular $r(B)$ can be calculated.

Using the form (2.12), it is shown in [28] that if $r(B)<1$ then the Green's function for $S$ can be written as

$$
G(t, s):=\left\langle(I-[B])^{-1} \mathcal{G}(s), \gamma(t)\right\rangle+G_{0}(t, s),
$$

where $\mathcal{G}(s)$ and $\gamma(t)$ denote vector functions with components $\mathcal{G}_{i}(s)$ and $\gamma_{i}(t)$, respectively and $\langle\cdot, \cdot\rangle$ stands for the inner product in $\mathbb{R}^{N}$.

It is also shown in [28] that if $f \geq 0$ then positive solutions do not exist if $B$ satisfies a positivity assumption, called $u_{0}$-positive (see for example [14]), and also $r([B])>1$. The case $r([B])=1$ is the resonant case.

Since $\gamma_{i}(t)>0$ on $(0,1)$, for an arbitrary subinterval $[a, b] \subset(0,1)$ there exist positive constants $c_{i}$ such that $\gamma_{i}(t) \geq c_{i}\left\|\gamma_{i}\right\|$ for $t \in[a, b]$. As shown in [28], it follows that if the Green's function of the local problem satisfies

$$
c_{0} \Phi_{0}(s) \leq G_{0}(t, s) \leq \Phi_{0}(s), t \in[a, b], s \in[0,1],
$$


then the Green's function for the non-local problem satisfies

$$
c \Phi(s) \leq G(t, s) \leq \Phi(s), t \in[a, b], s \in[0,1], \text { for } c=\min \left\{c_{0}, c_{1}, \ldots, c_{N}\right\}>0
$$

and a suitable $\Phi$. The authors of [28] were able to allow sign-changing measures by working in the associated cone

$$
K=\left\{u \in P: \min _{t \in[a, b]} u(t) \geq c\|u\|, \beta_{i}[u] \geq 0 \text { for every } i\right\} .
$$

From the above, we see that we first need to obtain properties for the Green's function $G_{0}$ of the local problem.

Thus, we begin by considering the local problem, (2.1) with the BCs

$$
u(0)=0, u^{\prime \prime}(0)=0, u(1)=0, u^{\prime \prime}(1)=0 .
$$

It is known and easy to see that the problem

$$
u^{(4)}(t)-\omega^{4} u(t)=f(t, u(t)), \quad u(0)=u^{\prime \prime}(0)=u(1)=u^{\prime \prime}(1)=0,
$$

is equivalent to the following BVP for the system of two second-order equations

$$
\begin{array}{ll}
-u^{\prime \prime}(t)-\omega^{2} u(t)=v(t), & u(0)=0, u(1)=0 ; \\
-v^{\prime \prime}(t)+\omega^{2} v(t)=f(t, u(t)), & v(0)=0, v(1)=0 .
\end{array}
$$

Therefore, the Green's function associated with (2.1)-(2.17) can be written as

$$
G_{0}(t, s)=\int_{0}^{1} G_{T}(t, \tau) G_{H}(\tau, s) d \tau
$$

where

$$
G_{T}(t, s):=\frac{1}{\omega \sin (\omega)} \begin{cases}\sin (\omega(1-t)) \sin (\omega s), & 0 \leq s \leq t \leq 1 \\ \sin (\omega t) \sin (\omega(1-s)), & 0 \leq t<s \leq 1\end{cases}
$$

and

$$
G_{H}(t, s):=\frac{1}{\omega \sinh (\omega)}\left\{\begin{array}{ll}
\sinh (\omega(1-t)) \sinh (\omega s), & 0 \leq s \leq t \leq 1 \\
\sinh (\omega t) \sinh (\omega(1-s)), & 0 \leq t<s \leq 1
\end{array} .\right.
$$

The subscripts $T, H$ indicate the involvement of trigonometric and hyperbolic functions. The Green's function can also be found by other calculations, the explicit expression is (see also [2])

$$
G_{0}(t, s)=\left\{\begin{array}{ll}
G_{1}(t, s):=\frac{\sin (\omega s) \sin (\omega(1-t))}{2 \omega^{3} \sin (\omega)}-\frac{\sinh (\omega s) \sinh (\omega(1-t))}{2 \omega^{3} \sinh (\omega)}, & s \leq t \\
G_{2}(t, s):=\frac{\sin (\omega t) \sin (\omega(1-s))}{2 \omega^{3} \sin (\omega)}-\frac{\sinh (\omega t) \sinh (\omega(1-s))}{2 \omega^{3} \sinh (\omega)}, & s>t
\end{array} .\right.
$$

Since we restrict $\omega$ to lie in $(0, \pi)$, we have $G_{0}(t, s) \geq 0$ for $t, s \in[0,1]$, and we note that the following symmetry properties hold:

$$
G_{1}(t, s)=G_{2}(s, t), \quad G_{1}(t, s)=G_{1}(1-s, 1-t), \quad G_{2}(t, s)=G_{2}(1-s, 1-t) .
$$


We will use the factored form (2.18) to show that $G_{0}$ satisfies $\left(C_{2}^{\prime}\right)$ for an explicit $c(t)$.

Firstly, as is known and readily shown, $\operatorname{since} \sinh (\omega t)$ is an increasing function of $t$, we have

$$
c_{H}(t) \Phi_{H}(s) \leq G_{H}(t, s) \leq \Phi_{H}(s), \quad \text { for } 0 \leq s, t \leq 1,
$$

where

$$
\Phi_{H}(s)=\frac{1}{\omega \sinh (\omega)} \sinh (\omega(1-s)) \sinh (\omega s)
$$

and

$$
c_{H}(t)=\min \left\{\frac{\sinh (\omega t)}{\sinh \omega}, \frac{\sinh (\omega(1-t))}{\sinh \omega}\right\} .
$$

When $0<\omega \leq \pi / 2$, a similar result holds for $G_{T}$

$$
c_{T}(t) \Phi_{T}(s) \leq G_{T}(t, s) \leq \Phi_{T}(s), \quad \text { for } 0 \leq s, t \leq 1,
$$

where

$$
\Phi_{T}(s)=\frac{1}{\omega \sin (\omega)} \sin (\omega(1-s)) \sin (\omega s)
$$

and

$$
c_{T}(t)=\min \left\{\frac{\sin (\omega t)}{\sin \omega}, \frac{\sin (\omega(1-t))}{\sin \omega}\right\} .
$$

When $\pi / 2<\omega<\pi$ the calculation is a little different, and since we have not seen this calculation written elsewhere, we give some details here.

LEMMA 2.1. For $\pi / 2<\omega<\pi$, the Green's function $G_{T}$ satisfies

$$
c_{T}(t) \Phi_{T}(s) \leq G_{T}(t, s) \leq \Phi_{T}(s), \text { for } 0 \leq s, t \leq 1,
$$

where

$$
\Phi_{T}(s)=\frac{1}{\omega \sin (\omega)} \begin{cases}\sin (\omega s), & s<1-\pi /(2 \omega) \\ \sin (\omega(1-s)) \sin (\omega s), & 1-\pi /(2 \omega) \leq s \leq \pi /(2 \omega), \\ \sin (\omega(1-s)), & s>\pi /(2 \omega)\end{cases}
$$

and

$$
c_{T}(t)=\min \{\sin (\omega t), \sin (\omega(1-t))\}
$$

Proof. The Green's function is

$$
G_{T}(t, s):=\left\{\begin{array}{ll}
G_{T 1}(t, s):=\frac{1}{\omega \sin (\omega)} \sin (\omega(1-t)) \sin (\omega s), & s \leq t \\
G_{T 2}(t, s):=\frac{1}{\omega \sin (\omega)} \sin (\omega t) \sin (\omega(1-s)), & s>t
\end{array} .\right.
$$


When $s \leq t \leq 1$, we have

$$
\sin (\omega(1-t)) \leq \begin{cases}1, & \text { if } s<1-\pi /(2 \omega) \\ \sin (\omega(1-s)), & \text { if } s \geq 1-\pi /(2 \omega)\end{cases}
$$

This shows that

$$
G_{T 1}(t, s) \leq \Phi_{T 1}(s):=\frac{1}{\omega \sin (\omega)} \begin{cases}\sin (\omega s), & s<1-\pi /(2 \omega) \\ \sin (\omega(1-s)) \sin (\omega s), & s \geq 1-\pi /(2 \omega)\end{cases}
$$

Similarly, arguments when $0 \leq t \leq s$ give

$$
G_{T 2}(t, s) \leq \Phi_{T 2}(s):=\frac{1}{\omega \sin (\omega)} \begin{cases}\sin (\omega(1-s)), & s>\pi /(2 \omega) \\ \sin (\omega(1-s)) \sin (\omega s), & s \leq \pi /(2 \omega)\end{cases}
$$

Thus, we have

$$
\begin{aligned}
\Phi_{T}(s) & := \begin{cases}\Phi_{T 1}(s), & s \leq 1 / 2 \\
\Phi_{T 2}(s), & s>1 / 2\end{cases} \\
& =\frac{1}{\omega \sin (\omega)} \begin{cases}\sin (\omega s), & s<1-\pi /(2 \omega) \\
\sin (\omega(1-s)) \sin (\omega s), & 1-\pi /(2 \omega) \leq s \leq \pi /(2 \omega) . \\
\sin (\omega(1-s)), & s>\pi /(2 \omega)\end{cases}
\end{aligned}
$$

We now determine $c(t)=c_{T}(t)$ so that $G_{T}(t, s) \geq c(t) \Phi_{T}(s)$. By the symmetry about $1 / 2$ in this problem it suffices to consider the case $t \leq 1 / 2$ and let $c(t):=c(1-t)$ for $t>1 / 2$.

There are then three regions to consider.

(1) $t \leq 1 / 2 \leq s \leq 1$, where we want to show $G_{T 2}(t, s) \geq c(t) \Phi_{T 2}(s)$.

(2) $t \leq s \leq 1 / 2$, where we want to show $G_{T 2}(t, s) \geq c(t) \Phi_{T 1}(s)$.

(3) $0 \leq s \leq t \leq 1 / 2$, where we want to show $G_{T 1}(t, s) \geq c(t) \Phi_{T 1}(s)$.

For region (1), we require $c(t)$ so that

$$
\sin (\omega t) \sin (\omega(1-s)) \geq c(t) \begin{cases}\sin (\omega(1-s)) \sin (\omega s), & s \leq \pi /(2 \omega) \\ \sin (\omega(1-s)), & s>\pi /(2 \omega)\end{cases}
$$

that is

$$
\frac{c(t)}{\sin (\omega t)} \leq \begin{cases}1 / \sin (\omega s), & s \leq \pi /(2 \omega) \\ 1, & s>\pi /(2 \omega)\end{cases}
$$

For this to hold for each $t$ and all appropriate $s$ we must have $c(t) \leq \sin (\omega t)$.

For region (2) where $t \leq s \leq 1 / 2$, we require $c(t)$ so that

$$
\sin (\omega t) \sin (\omega(1-s)) \geq c(t) \begin{cases}\sin (\omega s), & s \leq 1-\pi /(2 \omega) \\ \sin (\omega(1-s)) \sin (\omega s), & 1-\pi /(2 \omega)<s \leq 1 / 2\end{cases}
$$


that is

$$
\frac{c(t)}{\sin (\omega t)} \leq \begin{cases}\sin (\omega(1-s)) / \sin (\omega s), & t \leq s \leq 1-\pi /(2 \omega) \\ 1 / \sin (\omega s), & 1-\pi /(2 \omega)<s \leq 1 / 2\end{cases}
$$

Since $\omega s<\pi / 2$, both functions on the right side of (2.34) are decreasing functions of $s$, which coincide at $s=1-\pi /(2 \omega)$, so the minimum of the right side occurs when $s=1 / 2$ and so we need $c(t) \leq \frac{\sin (\omega t)}{\sin (\omega / 2)}$.

For region (3) a similar argument to that for region (1) gives $c(t) \leq \sin (\omega(1-t))$.

We have to take the minimum of all these possibilities, so the conclusion is

$$
c_{T}(t)=\min \{\sin (\omega t), \sin (\omega(1-t))\}
$$

which, by symmetry, is the formula for all $t \in[0,1]$ and is equivalent to

$$
c_{T}(t)=\left\{\begin{array}{ll}
\sin (\omega t), & \text { if } t \leq 1 / 2 \\
\sin (\omega(1-t)), & \text { if } t>1 / 2
\end{array} .\right.
$$

The following result is now immediate.

LEMMA 2.2. The Green's function for the local problem (2.1)-(2.17) satisfies

$$
c(t) \Phi_{0}(s) \leq G_{0}(t, s) \leq \Phi_{0}(s), \text { for } 0 \leq s, t \leq 1,
$$

where $c(t)=c_{T}(t), \Phi_{0}(s)=\int_{0}^{1} \Phi_{T}(\tau) G_{H}(\tau, s) d \tau$ and $\Phi_{T}, c_{T}$ are given by (2.26)-(2.27) for $0<\omega \leq \pi / 2$ and by (2.28)-(2.29) when $\pi / 2<\omega<\pi$.

REMARK 2.3. We could also write the Green's function as

$$
G_{0}(t, s)=\int_{0}^{1} G_{H}(t, \tau) G_{T}(\tau, s) d \tau,
$$

and expect to be able to use $c_{H}$ and $\Phi(s)=\int_{0}^{1} \Phi_{H}(\tau) G_{T}(\tau, s) d \tau$ as an alternative. Since, all other things being equal, choosing $c$ as large as possible so that Lemma 2.2 holds gives better results, we have chosen the given order because $c_{T}(t) \geq c_{H}(t)$ for every $\omega \in(0, \pi)$.

For a function $g$ that satisfies the Carathéodory conditions, we use the following notations:

$$
\begin{gathered}
\bar{g}(u):=\sup _{t \in[0,1]} g(t, u), \quad \underline{g}(u):=\inf _{t \in[0,1]} g(t, u), \\
g^{0}=\limsup _{u \rightarrow 0+} \bar{g}(u) / u, \quad g^{\infty}=\limsup _{u \rightarrow \infty} \bar{g}(u) / u, \\
g_{0}=\liminf _{u \rightarrow 0+} g(u) / u, \quad g_{\infty}=\liminf _{u \rightarrow \infty} \underline{g}(u) / u .
\end{gathered}
$$

For $r>0$ we also set

$$
g^{0, r}=\sup \{g(t, u) / r: t \in[0,1], u \in[0, r]\}
$$


and

$$
g_{r, r / c}=\inf \{g(t, u) / r: t \in[a, b], u \in[r, r / c]\} .
$$

We employ the constants $m$ and $M=M(a, b)$ defined by

$$
\frac{1}{m}=\sup _{t \in[0,1]} \int_{0}^{1} G(t, s) d s, \quad \frac{1}{M}=\inf _{t \in[a, b]} \int_{a}^{b} G(t, s) d s,
$$

and make use of the linear operator

$$
L u(t):=\int_{0}^{1} G(t, s) u(s) d s,
$$

where $G(t, s)$ is given by $(2.14)$.

Since $c(t) \Phi(s) \leq G(t, s) \leq \Phi(s)$, it follows that $L c(t) \geq\left(\int_{0}^{1} \Phi(s) c(s) d s\right) c(t)$, so by Krasnosel'skiü's Theorem 2.5 [13], $L$ has an eigenvalue $\lambda \geq \int_{0}^{1} \Phi(s) c(s) d s>0$, thus $r(L)>0$ and by the Krein-Rutman theorem, $r(L)$ is an eigenvalue of $L$ with a positive eigenfunction, the principal eigenvalue. Let $\mu_{1}=1 / r(L)$ be the principal characteristic value of $L$.

We recall the following theorem on the existence of multiple positive solutions for the following equation (fixed points of $S$ ), when $f \geq 0$ satisfies $\left(C_{3}\right)$, which is based on fixed point index results of [30], see Theorem 6.1 in [28],

$$
u(t)=\int_{0}^{1} G(t, s) f(s, u(s)) d s, \quad t \in[0,1] .
$$

We write $c_{[a, b]}=\min \{c(t): t \in[a, b]\}$.

TheOREM 2.4. Assume that $\left(C_{1}\right)-\left(C_{5}\right)$ hold and also that, whenever we have the condition $\mu_{1}<f_{\infty}$, we suppose $\left(C_{2}\right)$ holds for an arbitrary $[a, b] \subset(0,1)$. Then $(2.38)$ has a positive solution $u \in K$ if one of the following conditions holds.

$\left(S_{1}\right) 0 \leq f^{0}<\mu_{1}$ and $\mu_{1}<f_{\infty} \leq \infty$.

$\left(S_{2}\right) \mu_{1}<f_{0} \leq \infty$ and $0 \leq f^{\infty}<\mu_{1}$.

Equation (2.38) has at least two positive solutions in $K$ if one of the following conditions holds.

$\left(D_{1}\right) 0 \leq f^{0}<\mu_{1}, f_{r, r /\left[c_{[a, b]}\right.}>M$ for some $r>0$, and $0 \leq f^{\infty}<\mu_{1}$.

$\left(D_{2}\right) \mu_{1}<f_{0} \leq \infty, f^{0, r}<m$ for some $r>0$, and $\mu_{1}<f_{\infty} \leq \infty$.

Equation (2.38) has at least three positive solutions in $K$ if either $\left(T_{1}\right)$ or $\left(T_{2}\right)$ below holds.

$\left(T_{1}\right)$ There exist $0<r_{1}<c_{[a, b]} r_{2}<\infty$, such that

$$
0 \leq f^{0}<\mu_{1}, f_{r_{1}, r_{1} / c_{[a, b]}}>M, f^{0, r_{2}}<m, \quad \mu_{1}<f_{\infty} \leq \infty .
$$

$\left(T_{2}\right)$ There exist $0<r_{1}<r_{2}<\infty$, such that

$$
\mu_{1}<f_{0} \leq \infty, f^{0, r_{1}}<m, f_{r_{2}, r_{2} / c_{[a, b]}}>M, \quad 0 \leq f^{\infty}<\mu_{1} .
$$

REMARK 2.5. The list of conditions is readily extended to give a result on the existence of an arbitrary finite number of solutions under increasingly restrictive conditions on nonlinearity. The statements of these results are easily given, hence are omitted here. 
The restrictions on $f$ are weaker in $\left(D_{1}\right)$ if $[a, b]$ is chosen so that $M=M(a, b)$ is as small as possible: The height to be exceeded by the graph of $f$ is less. Also, for a given $[a, b]$ the restrictions on $f$ are weaker in $\left(D_{1}\right)$ and $\left(T_{1}\right)$ when $c$ is chosen as large as possible, since the length of interval on which $f$ has to be large is reduced. There is some interplay between these two requirements, the 'best' choice of $[a, b]$ would depend on the given nonlinearity $f$.

For the local problem, the 'optimal' interval, the one for which $M(a, b)$ is minimal, can be shown to be $[1 / 4,3 / 4]$.

There are also non-existence results, see $[\mathbf{2 5}, \mathbf{2 8}]$, which show that the hypotheses $\left(S_{1}\right)$ and $\left(S_{2}\right)$ in Theorem 2.4 are sharp.

THEOREM 2.6. The operator S has no non-zero fixed points in the cone $P$ if either $f(t, u)<\mu_{1} u$ for all $u>0$, or $f(t, u)>\mu_{1} u$ for all $u>0$.

We now give some examples of the values of $m, M(1 / 4,3 / 4), \mu_{1}, c_{[1 / 4,3 / 4]}$ for the local BVP

$$
u^{(4)}(t)-\omega^{4} u(t)=f(t, u(t)), \quad u(0)=0, u^{\prime \prime}(0)=0, u(1)=0, u^{\prime \prime}(1)=0,
$$

using the above formulas, where $c_{[1 / 4,3 / 4]}=\min \{c(t): t \in[1 / 4,3 / 4]\}$ with $c$ as in Lemma 2.2. These values of $c$ are not necessarily optimal. In particular, for $\omega=0$ a larger value has been found in [29] (with a different $\Phi(s)$ ) by another method. 0.2940

$$
\begin{aligned}
& \omega=0, m=384 / 5=76.8, M\left(\frac{1}{4}, \frac{3}{4}\right)=153.6, \mu_{1}=\pi^{4} \approx 97.4091, c_{\left[\frac{1}{4}, \frac{3}{4}\right]}=1 / 4 . \\
& \omega=1, \quad m \approx 76.0086, M\left(\frac{1}{4}, \frac{3}{4}\right) \approx 152.0172, \mu_{1}=\pi^{4}-\omega^{4} \approx 96.4091, c_{\left[\frac{1}{4}, \frac{3}{4}\right]} \approx \\
& 940 . \\
& \omega=\pi / 2, m \approx 71.9827, M\left(\frac{1}{4}, \frac{3}{4}\right) \approx 143.9657, \mu_{1} \approx 91.3210, c_{\left[\frac{1}{4}, \frac{3}{4}\right]} \approx 0.3827 . \\
& \omega=2, m \approx 64.1450, M\left(\frac{1}{4}, \frac{3}{4}\right) \approx 128.2899, \mu_{1} \approx 81.4091, c_{\left[\frac{1}{4}, \frac{3}{4}\right]} \approx 0.4794 . \\
& \omega=3, m \approx 12.8961, M\left(\frac{1}{4}, \frac{3}{4}\right) \approx 25.7922, \mu_{1} \approx 16.4091, c_{\left[\frac{1}{4}, \frac{3}{4}\right]} \approx 0.6816 .
\end{aligned}
$$

It is now routine to give examples of nonlinearities $f$ so that one, two, three or more positive solutions exist for the local problem. For the non-local problem it is necessary to calculate the constants, we shall give some examples later in the paper.

3. Resonant case. We now study the problem

$$
u^{(4)}(t)-\omega^{4} u(t)=f(t, u(t))
$$

subject to the BCs

$$
u(0)=\beta_{1}[u], \quad u^{\prime \prime}(0)+\beta_{2}[u]=0, \quad u(1)=\beta_{3}[u], \quad u^{\prime \prime}(1)+\beta_{4}[u]=0,
$$

when it is at resonance, that is when $r\left(\left[B_{\omega}\right]\right)=1$. It is to be noted that now we do not suppose that $f(t, u)$ is positive for all $u \geq 0$; see Theorem 3.4 for a good reason to not assume this. Instead, we assume that there exists $0<k<\omega$ such that $\widetilde{f}(t, u):=$ $f(t, u)+k^{4} u \geq 0$ for all $u \geq 0$.

Let $\widetilde{\omega}$ be defined by $\widetilde{\omega}^{4}=\omega^{4}-k^{4}$. Consider the equivalent shifted equation,

$$
u^{(4)}(t)-\widetilde{\omega}^{4} u(t)=\widetilde{f}(t, u(t)),
$$


where $\widetilde{f}(t, u):=f(t, u)+k^{4} u$, subject to the BCs (3.2). Let $\widetilde{\gamma}_{j}$ be the solutions of

$$
\widetilde{\gamma}^{(4)}(t)-\widetilde{\omega}^{4} \widetilde{\gamma}(t)=0
$$

under the respective BCs (2.7), (2.8), (2.9) and (2.10). Thus, these are given by

$$
\begin{aligned}
& \widetilde{\gamma}_{1}(t)=\sin (\widetilde{\omega}(1-t)) /(2 \sin (\widetilde{\omega}))+\sinh (\widetilde{\omega}(1-t)) /(2 \sinh (\widetilde{\omega})), \\
& \widetilde{\gamma}_{2}(t)=\sin (\widetilde{\omega}(1-t)) /\left(2 \widetilde{\omega}^{2} \sin (\widetilde{\omega})\right)-\sinh (\widetilde{\omega}(1-t)) /\left(2 \widetilde{\omega}^{2} \sinh (\widetilde{\omega})\right), \\
& \widetilde{\gamma}_{3}(t)=\sin (\widetilde{\omega} t) /(2 \sin (\widetilde{\omega}))+\sinh (\widetilde{\omega} t) /(2 \sinh (\widetilde{\omega})), \\
& \widetilde{\gamma}_{4}(t)=\sin (\widetilde{\omega} t) /\left(2 \widetilde{\omega}^{2} \sin (\widetilde{\omega})\right)-\sinh (\widetilde{\omega} t) /\left(2 \widetilde{\omega}^{2} \sinh (\widetilde{\omega})\right) .
\end{aligned}
$$

The shifted problem (3.3)-(3.2) is not at resonance when $r\left(\left[B_{\widetilde{\omega}}\right]\right)<1$, where $\left[B_{\widetilde{\omega}}\right]$ is the $N \times N$ matrix with entries $\beta_{i}\left[\widetilde{\gamma}_{j}\right]$, and we suppose that $\beta_{i}\left[\widetilde{\gamma}_{j}\right] \geq 0$.

In order to show that the shifted problem is not at resonance the following result will be useful.

LEMMA 3.1. Each $\gamma_{i}$ is a strictly increasing function of $\omega$.

Proof. Let $\omega \in(0, \pi)$ and let $G_{0}$ be the Green's function for the problem

$$
u^{(4)}(t)-\omega^{4} u(t)=y(t), u(0)=0, u^{\prime \prime}(0)=0, u(1)=0, u^{\prime \prime}(1)=0,
$$

as in (2.18) or (2.21). Then $G_{0}(t, s)>0$ for $t, s \in(0,1)$.

Let $\gamma_{1}(\omega)$ be the solution of

$$
\gamma^{(4)}(t)-\omega^{4} \gamma(t)=0
$$

satisfying the BCs

$$
\gamma(0)=1, \gamma^{\prime \prime}(0)=0, \gamma(1)=0, \gamma^{\prime \prime}(1)=0 .
$$

Then, see (2.11), $\gamma_{1}(\omega)>0$ is strictly positive on $(0,1)$. Let $v_{1}=\gamma_{1}\left(\omega_{1}\right)$ and $v_{2}=\gamma_{1}\left(\omega_{2}\right)$ denote the solutions of (3.5) and (3.6) with $\omega=\omega_{1}$ and $\omega=\omega_{2}$, respectively. Assume $\omega_{2}>\omega_{1}$. Then $v:=v_{2}-v_{1}$ satisfies the equation

$$
v^{(4)}(t)-\omega_{1}^{4} v(t)=\left(\omega_{2}^{4}-\omega_{1}^{4}\right) v_{2}(t)
$$

with BCs

$$
v(0)=0, v^{\prime \prime}(0)=0, v(1)=0, v^{\prime \prime}(1)=0 .
$$

Thus,

$$
v(t)=\int_{0}^{1} G_{0}(t, s)\left(\omega_{2}^{4}-\omega_{1}^{4}\right) v_{2}(s) d s>0, \quad \text { for } t \in(0,1) .
$$

This proves that $\omega_{2}>\omega_{1}$ implies $v_{2}(t)>v_{1}(t)$ for $t \in(0,1)$, that is $\gamma_{1}$ is a strictly increasing function of $\omega$.

An exactly similar argument shows that the same holds for each of $\gamma_{2}, \gamma_{3}, \gamma_{4}$.

THEOREM 3.2. Suppose that for some $k \in(0, \omega)$ we have $\widetilde{f}(t, u):=f(t, u)+k^{4} u \geq 0$ for all $u \geq 0$. Let $\widetilde{\omega}^{4}=\omega^{4}-k^{4}$ and suppose that $\beta_{i}\left[\gamma_{j}\right] \geq 0$ and $r\left(\left[B_{\omega}\right]\right)=1$, and that 
$\beta_{i}\left[\widetilde{\gamma}_{j}\right] \geq 0$ and $r\left(\left[B_{\widetilde{\omega}}\right]\right)<1$. Moreover, suppose that $\left(C_{4}\right)$ holds. Then the resonant problem (3.1)-(3.2) has at least one positive solution if one of $(\mathrm{Sa})$ or $(\mathrm{Sb})$ holds, where

(Sa) $f^{0}<0$ and $f_{\infty}>0$,

(Sb) $f_{0}>0$ and $f^{\infty}<0$.

The resonant problem (3.1)-(3.2) has at least two positive solutions if either of the following conditions hold.

(Da) $f^{0}<0$, and $f^{\infty}<0$, and there exists

$r>0$ such that $\inf _{t \in[a, b]} f(t, u)+k^{4} u>r \tilde{M}$ for $u \in[r, r \widetilde{c}]$.

(Db) $f_{0}>0$, and $f_{\infty}>0$, and there exists

$r>0$ such that $\sup _{t \in[0,1]} f(t, u)+k^{4} u<r \tilde{m}$ for $u \in[0, r]$.

Here $\widetilde{c}=\min \left\{\widetilde{c}_{0}, \widetilde{c}_{1}, \ldots, \widetilde{c}_{N}\right\}$ and $\tilde{m}, \tilde{M}$ are the constants defined in (2.15) and (2.36), respectively, for the shifted problem

$$
u^{(4)}(t)-\widetilde{\omega}^{4} u(t)=\tilde{f}(t, u(t))
$$

with the given BCS.

Proof. Let $\tilde{\mu}_{1}$ denote the principal characteristic value corresponding to (3.3) with the BCs (3.2). Since the problem is at resonance, we have $\mu_{1}=0$. Clearly $\tilde{\mu}_{1}=$ $\mu_{1}+k^{4}=k^{4}$. Since $\widetilde{f}^{0}=f^{0}+k^{4}$, with similar expressions for the other terms, $(S a)$ implies $0 \leq \widetilde{f}^{0}<\widetilde{\mu}_{1}$ and $\widetilde{\mu}_{1}<\widetilde{f}_{\infty} \leq \infty$. This means that $\left(S_{1}\right)$ of Theorem 2.4 holds for $\widetilde{f}$. Similarly $\left(S_{2}\right)$ holds for $\widetilde{f}$ when $(S b)$ holds for $f$. Hence, the resonant problem (3.1)-(3.2) has at least one positive solution. Similarly $(D a)$ and $(D b)$ holding for $f$ imply that $\left(D_{1}\right)$ and $\left(D_{2}\right)$ hold for $\widetilde{f}$.

REMARK 3.3. Similarly one can state a result for three or any finite number of positive solutions using Theorem 2.4.

When $\beta_{i}$ are positive functionals then by Lemma 3.1 we have $0<\beta_{i}\left[\tilde{\gamma}_{j}\right]<\beta_{i}\left[\gamma_{j}\right]$, since $\widetilde{\omega}<\omega$. As $\left[B_{\widetilde{\omega}}\right],\left[B_{\omega}\right]$ are positive matrices, it follows that $0<r\left(\left[B_{\widetilde{\omega}}\right]\right)<r\left(\left[B_{\omega}\right]\right)=$ 1 , so the perturbed problem is not at resonance in this case for any $k \in(0, \omega)$. In fact this remains valid if $\left[B_{\widetilde{\omega}}\right]$ is a non-negative irreducible matrix, see, for example [22, 23].

THEOREM 3.4. The resonant problem (3.1)-(3.2) has no positive solutions if either

$$
f(t, u)<0 \text { for all } u>0 \text {, or } f(t, u)>0 \text { for all } u>0 .
$$

Proof. For example, if $f(t, u)>0$ for all $u>0$, then $\tilde{f}(t, u)=f(t, u)+k^{4} u>k^{4} u=$ $\tilde{\mu}_{1} u$ and Theorem 2.6 applies.

This means that some change of sign is necessary to have positive solution in the resonance case; this is usually derived as a consequence of the Fredholm alternative. Some second-order problems were discussed in [24], where a necessary and sufficient condition was given for the existence of a positive solution when $f$ is non-negative (or non-positive).

4. Examples. We now give some examples; because we use a shift argument, these are examples of both the resonant and non-resonant cases at once. To see examples with sign-changing measures for some second-order problems, see [26, 27]. Here we could give similar examples but have concentrated on simple new examples to illustrate the approach using a shift argument. 
EXAMPLE 4.1. An example with four point mass functionals acting at a single point.

Consider the equation $u^{(4)}(t)-\omega^{4} u(t)=f(u(t))$, that is (2.1) with $0<\omega<\pi$, subject to the BCs

$$
u(0)=\beta_{1} u(\eta), \quad u^{\prime \prime}(0)+\beta_{2} u(\eta)=0, \quad u(1)=\beta_{3} u(\eta), \quad u^{\prime \prime}(1)+\beta_{4} u(\eta)=0,
$$

where $\beta_{i}>0$ and $\eta \in(0,1)$. The same BCs were considered in [28] in the non-resonant case. In this case

$$
[B]:=\left[\begin{array}{llll}
\beta_{1} \gamma_{1}(\eta) & \beta_{1} \gamma_{2}(\eta) & \beta_{1} \gamma_{3}(\eta) & \beta_{1} \gamma_{4}(\eta) \\
\beta_{2} \gamma_{1}(\eta) & \beta_{2} \gamma_{2}(\eta) & \beta_{2} \gamma_{3}(\eta) & \beta_{2} \gamma_{4}(\eta) \\
\beta_{3} \gamma_{1}(\eta) & \beta_{3} \gamma_{2}(\eta) & \beta_{3} \gamma_{3}(\eta) & \beta_{3} \gamma_{4}(\eta) \\
\beta_{4} \gamma_{1}(\eta) & \beta_{4} \gamma_{2}(\eta) & \beta_{4} \gamma_{3}(\eta) & \beta_{4} \gamma_{4}(\eta)
\end{array}\right]
$$

and the eigenvalues are $0,0,0, \sum_{i=1}^{4} \beta_{i} \gamma_{i}(\eta)$ (note that $[B]$ has rank 1 ), thus

$$
r([B])=\sum_{i=1}^{4} \beta_{i} \gamma_{i}(\eta) .
$$

If $r([B])=1$, the problem $(2.1)-(4.1)$ is at resonance. Consider the shifted equation

$$
u^{(4)}(t)-\widetilde{\omega}^{4} u(t):=u^{(4)}(t)-\left(\omega^{4}-k^{4}\right) u(t)=f(t, u(t))+k^{4} u(t),
$$

with $0<k<\omega$ subject to the same BCs. Note that $\beta_{i} \tilde{\gamma}_{i}(\eta)>0$ since $\tilde{\gamma}_{i}>0$ on $(0,1)$. By Lemma 3.1, the shifted problem is non-resonant for any $k$ with $0<k<\omega$ and we can choose any such $k$ for which $f$ satisfies $f(t, u)+k^{4} u \geq 0$ for all $u \geq 0$.

We now give a specific example. Let $f(u):=\frac{u(-1+3 u)}{16+u^{1 / 2}+4 u}$ and consider the problem

$$
u^{(4)}(t)-u(t)=f(u(t)), \quad t \in(0,1),
$$

where we have $\omega=1$, with the BCs

$$
u(0)=\beta_{1} u(1 / 2), \quad u^{\prime \prime}(0)+\beta_{2} u(1 / 2)=0, \quad u(1)=\beta_{3} u(1 / 2), \quad u^{\prime \prime}(1)+\beta_{4} u(1 / 2)=0,
$$

and, with $p=\sin (1) / \sin (1 / 2)$, take $\beta_{1}=p / 2, \beta_{2}=p / 4, \beta_{3}=p / 2, \beta_{4}=3 p / 4$. Then, by calculation, $r([B])=\sum_{i=1}^{4} \beta_{i} \gamma_{i}(\eta)=1$.

We may choose any $k \in[1 / 2,1)$. Then $f_{0}=-1 / 16$ and $f^{\infty}=3 / 4$ so by Theorem 3.2, case $(S b)$, there exists one positive solution.

EXAMPLE 4.2. Consider the equation

$$
u^{(4)}(t)-\omega^{4} u(t)=f(u(t)), \quad t \in(0,1),
$$

for $0<\omega<\pi$ with two local BCs and two functional BCs

$$
u(0)=\beta_{1}[u], \quad u^{\prime \prime}(0)=0, \quad u(1)=\beta_{3}[u], \quad u^{\prime \prime}(1)=0 .
$$

Then

$$
[B]:=\left[\begin{array}{ll}
\beta_{1}\left[\gamma_{1}\right] & \beta_{1}\left[\gamma_{3}\right] \\
\beta_{3}\left[\gamma_{1}\right] & \beta_{3}\left[\gamma_{3}\right]
\end{array}\right],
$$


where

$$
\begin{aligned}
& \gamma_{1}(t)=\sin (\omega(1-t)) /(2 \sin (\omega))+\sinh (\omega(1-t)) /(2 \sinh (\omega)), \\
& \gamma_{3}(t)=\sin (\omega t) /(2 \sin (\omega))+\sinh (\omega t) /(2 \sinh (\omega)) .
\end{aligned}
$$

For a specific example we take BCs of integral type $\beta_{j}[u]=\beta_{j} \int_{0}^{1} u(t) d t$ where $\beta_{j} \geq 0$. For this choice of $\mathrm{BCs}, r([B])=\beta_{1}\left[\gamma_{1}\right]+\beta_{3}\left[\gamma_{3}\right]$, which explicitly is

$$
r([B])=\left(\beta_{1}+\beta_{3}\right)(\sin (\omega)(\cosh (\omega)-1)+\sinh (\omega)(1-\cos (\omega))) /(2 \omega \sin (\omega) \sinh (\omega)) .
$$

Thus, for $\beta_{1}+\beta_{3}=2 \omega \sin (\omega) \sinh (\omega) /(\sin (\omega)(\cosh (\omega)-1)+\sinh (\omega)(1-\cos (\omega)))$ the problem is at resonance. For example, when $\omega=1$, we can have $\beta_{1}=1, \beta_{3} \approx 0.9833$. We now choose and fix these values of $\omega, \beta_{1}, \beta_{3}$. Consider the nonlinearity defined by

$$
f(u)=\left(u^{1 / 2}+u^{2}\right) / 3-3 \sin (u) / 4 .
$$

Then $f(u)+k^{4} u \geq 0$ for $k^{4} \geq 3 / 4$. Also, $f_{0}=\infty, f_{\infty}=\infty$, so Theorem $3.2(D b)$ will apply if we can show there exist $r$ and $\widetilde{\omega}$ such that $f(u)+\left(\omega^{4}-\widetilde{\omega}^{4}\right) u \leq \tilde{m} r$ for $0 \leq u \leq r$. Choose $k^{4}=3 / 4$ and $\widetilde{\omega}=1 / \sqrt{2}$, and we want

$$
f(u)+3 u / 4 \leq \tilde{m} r, \text { for } 0 \leq u \leq r .
$$

By a calculation with Maple, $\tilde{m} \approx 0.74717$. Since $f(u)+3 u / 4$ is increasing, it suffices to have $r$ such that

$$
f(r) / r+3 / 4 \leq \tilde{m}
$$

Recall that $f$ takes some negative values. This is satisfied when $0.2620<r<0.8958$, in particular we may take $r=1 / 2$. Thus, this problem has two positive solutions.

\section{REFERENCES}

1. Z. Bai, W. Li and W. Ge, Existence and multiplicity of solutions for four-point boundary value problems at resonance, Nonlinear Anal. 60 (2005), 1151-1162.

2. A. Cabada, J. A. Cid and L. Sanchez, Positivity and lower and upper solutions for fourth-order boundary value problems, Nonlinear Anal. 67 (2007), 1599-1612.

3. A. Cabada and R. R. Enguiça, Positive solutions of fourth-order problems with clamped beam boundary conditions, Nonlinear Anal. 74 (2011), 3112-3122.

4. J. A. Cid, D. Franco and F. Minhos, Positive fixed points and fourth-order equations, Bull. Lond. Math. Soc. 41 (2009), 72-78.

5. W. Feng and J. R. L. Webb, Solvability of three-point boundary value problems at resonance, Nonlinear Anal. 30 (1997), 3227-3238.

6. C. P. Gupta, Existence theorems for a second-order $m$-point boundary value problem at resonance, Int. J. Math. Math. Sci. 18 (1995), 705-710.

7. X. Han, Positive solutions for a three-point boundary value problem at resonance, J. Math. Anal. Appl. 336 (2007), 556-568.

8. G. Infante and P. Pietramala, A cantilever equation with nonlinear boundary conditions, Electron. J. Qual. Theory Differ. Equ. Special Edition I(15) (2009), 14 pp.

9. G. Infante, P. Pietramala and M. Zima, Positive solutions for a class of nonlocal impulsive BVPs via fixed point index, Topol. Methods Nonlinear Anal. 36 (2010), 263-284.

10. G. Infante and M. Zima, Positive solutions of multi-point boundary value problems at resonance, Nonlinear Anal. 69 (2008), 2458-2465. 
11. N. Kosmatov, A symmetric solution of a multipoint boundary value problem at resonance, Abstr. Appl. Anal. Art. ID 54121 (2006), 11 pp.

12. N. Kosmatov, Multi-point boundary value problems on an unbounded domain at resonance, Nonlinear Anal. 68 (2008), 2158-2171.

13. M. A. Krasnosel'ski1, Positive solutions of operator equations (P. Noordhoff, Groningen, Netherlands, 1964).

14. M. A. Krasnosel'skiĭ and P. P. Zabreǐko, Geometrical methods of nonlinear analysis (Springer-Verlag, Berlin, 1984).

15. K. Q. Lan and J. R. L. Webb, Positive solutions of semilinear differential equations with singularities, J. Differ. Equ. 148 (1998), 407-421.

16. B. Liu, Solvability of multi-point boundary value problem at resonance, IV, Appl. Math. Comput. 143 (2003), 275-299.

17. R. Ma, Existence results of a $m$-point boundary value problem at resonance, J. Math. Anal. Appl. 294 (2004), 147-157.

18. R. Ma and T. Chen, Existence of positive solutions of fourth-order problems with integral boundary conditions, Bound. Value Probl. Art. ID 297578 (2011), 17 pp.

19. R. Ma and Y. Yang, Existence result for a singular nonlinear boundary value problem at resonance, Nonlinear Anal. 68 (2008), 671-680.

20. J. Mawhin, Equivalence theorems for nonlinear operator equations and coincidence degree theory for mappings in locally convex topological vector spaces, J. Differ. Equ. 12 (1972), 610-636.

21. D. O'Regan and M. Zima, Leggett-Williams norm-type theorems for coincidences, Arch. Math. 87 (2006), 233-244.

22. E. Seneta, Non-negative matrices and Markov chains (revised reprint of the second (1981) edn.), Springer Series in Statistics (Springer, New York, 2006).

23. R. S. Varga, Matrix iterative analysis (Prentice-Hall, New Jersey, 1963) (second printing).

24. J. R. L. Webb, Remarks on non-local boundary value problems at resonance, Appl. Math. Comput. 216 (2010), 497-500.

25. J. R. L. Webb, Solutions of nonlinear equations in cones and positive linear operators, J. Lond. Math. Soc. 82(2) (2010), 420-436.

26. J. R. L. Webb and G. Infante, Positive solutions of nonlocal boundary value problems: A unified approach, J. Lond. Math. Soc. 74(2) (2006), 673-693.

27. J. R. L. Webb and G. Infante, Positive solutions of nonlocal boundary value problems involving integral conditions, Nonlinear Differ. Equ. Appl. (NoDEA) 15 (2008), 45-67.

28. J. R. L. Webb and G. Infante, Non-local boundary value problems of arbitrary order, J. Lond. Math. Soc. 79(2) (2009), 238-258.

29. J. R. L. Webb, G. Infante and D. Franco, Positive solutions of nonlinear fourthorder boundary-value problems with local and non-local boundary conditions, Proc. Roy. Soc. Edinburgh A 138 (2008), 427-446.

30. J. R. L. Webb and K. Q. Lan, Eigenvalue criteria for existence of multiple positive solutions of nonlinear boundary value problems of local and nonlocal type, Topol. Methods Nonlinear Anal. 27 (2006), 91-116.

31. J. R. L. Webb and M. Zima, Multiple positive solutions of resonant and non-resonant nonlocal boundary value problems, Nonlinear Anal. 71 (2009), 1369-1378. 